\author{
Cadernos de \\ ESTUDOS LINGḯlSTICOS - (57.1), Campinas, Jan./Jun. 2015
}

\title{
EXPLETIVOS NULOS E CONSTRUÇÕES DE TÓPICO/SUJEITO NO PORTUGUES BRASILEIRO
}

\author{
MARY A. KATO \\ UNICAMP/CNPQ
}

\begin{abstract}
RESUMO: Apesar de o Português Brasileiro (PB) estar perdendo o sujeito nulo referencial desde o século passado, o expletivo nulo vem sendo mantido ( $\varnothing$ chove muito nessas florestas), apesar de línguas como o francês e o espanhol dominicano terem desenvolvido expletivos lexicais ao lado de sujeitos pronominais referenciais. O que vem sendo notado, entretanto, é que o PB vem desenvolvendo construções pessoais com o sujeito lexical movido por alçamento (Essas florestas chovem muito), aos quais vimos chamando de tópico-sujeito. O presente trabalho descarta duas hipóteses anteriores sobre a sobrevivência das construções com expletivo nulo e defende que as duas construções devem co-existir por não constituirem formas em competição ("doublets"no sentido de Kroch ( 1994)), sendo a primeira uma construção tética e a segunda uma construção categórica. Estas substituem outras construções categóricas mais antigas, de redobro clítico.
\end{abstract}

\begin{abstract}
Though Brazilian Portuguese has been losing its null referential subjects, its null expletive ( $\varnothing$ chove muito nessas florestas) has been maintained, though other languages that have undergone similar changes in the domain of referential null subjects have equally lost the null expletive, and developed lexical expletives (Dominican Spanish: Ello quiere llover.) What has been observed, however, is that BP has been developing constructions with lexical subjects resulted from raising, which we have been calling topic-subject constructions. The present study abandons two former hypothesis about the possible development of lexical expletives, considering that the two present constructions (one with a null expletive and the other with a topic-subject ) do not constitute real competitors, since the former is a thetic structure and the latter is a categorical one. The paper also defends that the latter is a modern development of the old CLLD structure, which is also of a categorical nature.
\end{abstract}

\section{INTRODUÇÃO}

O Português Brasileiro (PB) vem sofrendo uma série de mudanças desde o século XIX, entre as quais, a perda do sujeito nulo referencial, a perda da inversão livre (VXS) e a perda de subida longa do clítico, o que pode ser interpretado como uma mudança de ordem paramétrica, ou mudança no valor do Parâmetro do Sujeito Nulo (Duarte 1995; Figueiredo Silva 1996; Kato 1999, entre outros).

Assim, durante todo o século XIX, os sujeitos referenciais de primeira, segunda e terceira pessoas eram preferencialmente nulos como mostra Duarte (1993, 1995), em exemplos como (1): 
(1) a. Quando ( ) te vi pela primeira vez, () não sabia que () eras viúva e rica. (1845)

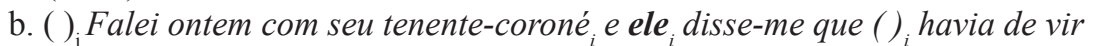
com sinhá Dona Perpétua e com sinhá moça Rosinha. (1882)

Já no século XX, o que temos é o sujeito preferencialmente expresso, como mostram

Duarte (1995) e Duarte \& Kato (2014):

(2) a. Eu acho que eu passaria por causa da base que eu tinha.

b. Vocês dizem isso porque vocês são jovens.

c. Ela ficou solteira porque ela quis.

Duarte (1993) mostra que o preenchimento do sujeito pronominal é da ordem de apenas $20 \%$ na segunda metade do século XIX (80\% de nulos) chegando a quase $80 \%$ no final do século XX (16\% de nulos), considerando juntas as três pessoas do discurso.

Tabela 1 : Distribuição dos sujeitos de $1^{\mathrm{a}}$., $2^{\mathrm{a}}$. e $3^{\mathrm{a}}$, pessoas nulos (vs expressos) (Duarte (1993)

\begin{tabular}{|c|c|c|c|}
\hline Período & $\mathbf{1}^{\text {a }}$.pessoa & $\mathbf{2}^{\text {a }}$. pessoa & $\mathbf{3}^{\text {a }}$.pessoa \\
\hline Período I & $50 / 72$ & $37 / 38$ & $33 / 40$ \\
1845 & $\mathbf{6 9 \%}$ & $\mathbf{9 7 \%}$ & $\mathbf{8 3 \%}$ \\
\hline Período II & $50 / 63$ & $50 / 63$ & $16 / 24$ \\
1889 & $\mathbf{7 9 \%}$ & $\mathbf{7 9 \%}$ & $\mathbf{6 7 \%}$ \\
\hline Período III & $54 / 65$ & $32 / 45$ & $27 / 41$ \\
1919 & $\mathbf{8 3 \%}$ & $\mathbf{7 1 \%}$ & $\mathbf{6 6 \%}$ \\
\hline Período IV & $43 / 71$ & $10 / 40$ & $28 / 39$ \\
1937 & $\mathbf{6 1 \%}$ & $\mathbf{2 5 \%}$ & $\mathbf{7 2 \%}$ \\
\hline Período V & $46 / 82$ & $6 / 29$ & $23 / 39$ \\
1955 & $\mathbf{5 6 \%}$ & $\mathbf{2 1 \%}$ & $\mathbf{5 9 \%}$ \\
\hline Período VI & $25 / 79$ & $8 / 40$ & $16 / 31$ \\
1975 & $\mathbf{3 2 \%}$ & $\mathbf{2 0 \%}$ & $\mathbf{5 2 \%}$ \\
\hline Período VII & $18 / 99$ & $14 / 63$ & $21 / 38$ \\
1992 & $\mathbf{1 8 \%}$ & $\mathbf{2 2 \%}$ & $\mathbf{5 5 \%}$ \\
\hline
\end{tabular}

Além da mudança quantitativamente significativa dessa propriedade, o que é surpreendente são as mudanças correlacionadas, tais como ordem sintática. Assim. Berlinck (1995), Kato e Tarallo (2003) e Kato et alii (2006) mostraram que o PB vem perdendo a inversão livre no mesmo período, com verbos transitivos exibindo uma perda quase categórica. 
Cadernos de ESTUDOS LINGḯISTICOS (57.1) - Jan./Jun. 2015

(3) a. Moram comigo dois dos meus filhos. (século XIX)

b. Dois dos meus filhos moram comigo.

(século XX)

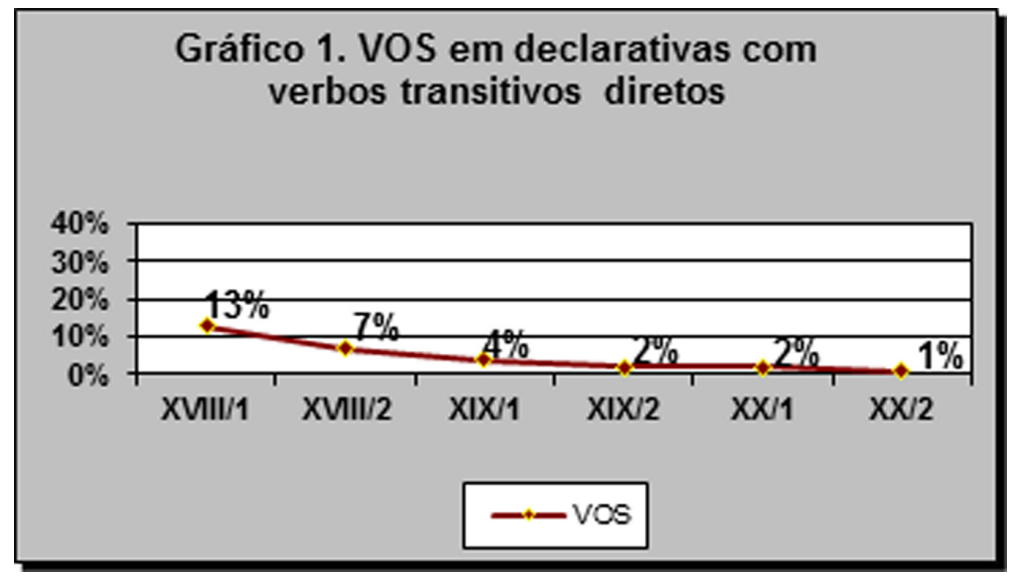

Outra propriedade também atribuída ao Parâmetro do Sujeito Nulo (Kayne 1989) é a subida longa do clítico, que também mostra mudança, segundo Pagotto (1993) e Cyrino (1993).

(4) a.João não me tinha cumprimentado. (século XIX)

b. João não tinha me cumprimentado (século XX)

Comparando as mudanças, Kato (2012) verifica que elas ocorrem paralelamente, sugerindo uma mudança de ordem paramétrica.

\begin{tabular}{|c|c|c|c|}
\hline PB & +Sujeito Nulo & VOS & +Subida do clítico \\
\hline $\begin{array}{c}2^{\text {a. }} \text { metade do século } \\
\text { XIX }\end{array}$ & $80 \%$ & $13 \%$ & $100 \%$ \\
\hline $2^{\text {a }}$ metade de XX & $16 \%$ & $1 \%$ & $\varnothing \%$ \\
\hline
\end{tabular}

Tabela 1: mudanças paralelas desde o século XIX

Verifica-se que, enquanto a perda com relação à subida dos clíticos é categórica e a perda da ordem VOS é quase categórica, a perda do sujeito nulo revela contextos de resistência à mudança.

Assim, comparado o PB a uma língua como o Espanhol Dominicano (ED), que vem sofrendo mudanças similares ao PB, Kato e Duarte (2014) verificaram que, em construções impessoais, aquela vem preenchendo o sujeito com um expletivo pronominal (cf. Toribio 1996), enquanto o PB mantém o expletivo nulo como um contexto de resistência. 
(5) a. Ello quiere llover.

b. Ello hay muchos mangoes in Bahia.

c. Ello parece que no hay azúcar.

(Toribio 1996)

(6) a. Øexpl Está querendo chover.

b. Øexpl Tem muitas mangas na Bahia.

c. Øexpl Parece que não tem açúcar. （Kato \&Duarte 2008)

\section{DUAS HIPÓTESES PRELIMINARES PARA EXPLICAR A DIFERENÇA ENTRE O ED E O PB}

\subsection{A hipótese da hierarquia de referencialidade na mudança linguística}

Cyrino, Duarte e Kato (2000) propõem que a referencialidade tem uma relevância altamente translinguística na pronominalização. Por outro lado, para uma língua que tem uma opção interna para variantes nulas ou não-nulas, um fator forte para a seleção de uma forma ou outra é o estatuto referencial do antecedente.

Na hipótese das autoras, argumentos [+N, +humano] estão no extremo mais alto na hierarquia referencial, enquanto não-argumentos estão na posição mais baixa. Com relação aos pronomes, o falante (eu) e o interlocutor (você), sendo inerentemente humanos, primeira e segunda pessoas pronominais, estão no ponto mais alto na hierarquia, e o pronome de terceira pessoa que não é argumental está na posição mais baixa, com a entidade 3a pessoa [-humana] em posição intermediária. $\mathrm{O}$ traço $[ \pm$ específico] interage com todos esses traços:

I. Hierarquia Referencial

não-argumento proposição [-humano $]$ [+humano $]$

3 p. $\quad 3$ p. 2 p. 1 p.

-espec. +espec.

$[-$ ref $]<-$

Propõe-se, a partir dessa generalização, a seguinte hipótese:

II. Hipótese do Mapeamento Implicacional

a. quanto mais referencial, maior a possibilidade de um pronome não-nulo;

b. uma variante nula em um ponto específico da escala implica uma variante nula à sua esquerda, na hierarquia referencial.

Relativamente ao nosso problema de mudança, da perda do sujeito nulo no $\mathrm{PB}$, parece haver efetivamente uma aplicação plausível dessa hierarquia uma vez 
que a primeira e segunda pessoa são as primeiras pessoas a perderem o nulo e o elemento não-argumental (expletivo) ainda preserva o nulo, com a terceira pessoa, no meio, ainda em variação.

\subsection{Inovações nas construções impessoais (segunda hipótese)}

Pesquisas mais recentes, entretanto, revelam que a posição de sujeito não referencial pode aparecer foneticamente realizada. Ao contrário, porém, de desenvolver um expletivo lexical, como ocorreu no francês antigo, com o aparecimento do expletivo $i l$, e como ocorre no presente, com uma variedade do espanhol da República Dominicana, que, segundo Toribio (1996), desenvolveu o expletivo "ello", o PB, além de manter o expletivo nulo, lança mão de elementos pessoais para ocupar a posição de sujeito através do alçamento do locativo argumental de sentenças existenciais (7a), o alçamento do sujeito de completivas de verbos do tipo custar, demorar, etc (8b), ou ainda de um argumento interno a DP de verbos inacusativos (9c):

(7) a. Tem prédios lindos em Londres

b. Londres ${ }_{i}$ tem prédios lindos $\left[t_{i}\right]$.

(8) a. Custou pra ele sair de casa.

b. Ele ${ }_{i}$ custou [a $t_{i}$ sair de casa].

(9) a. Furou o pneu do carro/

b. $\left[\boldsymbol{O}\right.$ carro $_{i}$ furou [o pneu $\left.\left[t_{i}\right]\right]$

Kato e Duarte (2008) recuperam a hipótese de Pontes (1989) de que o PB estaria mudando de uma língua de proeminência de sujeito para uma língua de proeminência de tópico ${ }^{1}$ e de sujeito. ${ }^{2}$ As autoras justificam essa hipótese alegando que línguas de proeminência de tópico não contam com expletivos lexicais e que a satisfação do EPP nesse tipo de línguas se daria via alçamento. Com efeito, uma língua de proeminência de tópico como o japonês não conta com expletivos nulos, mas conta com construções de alçamento, marcadas com -wa análogas ao PB:

(10) a. London-wa utsukushii biru ga

aru.

Londres-top bonito predio-nom tem

b.Kare-wa uti-kara deruno-ni hima-dotta. ele-top casa-de sair-em tempo-levou

c. Kuruma-wa taya-ga panku-shita. carro-top pneu-nom. furar-passado

\footnotetext{
${ }^{1}$ Esta é a nomenclatura funcionalista de Li e Thompson (1976) equivalente a "línguas de proeminência discursiva" da gerativista Kiss (1995).

${ }^{2}$ Outros autores vêm trabalhando em hipóteses semelhantes, como por exemplo Negrão (1999), e.o.
} 
$\mathrm{Na}$ presente seção, procurarei defender essa proposta usando o modelo de Miyagawa (2010, 2007), conhecido como o "feature inheritance approach" (modelo de herança de traços) ${ }^{3}$.

Segundo Chomsky (2008), os traços-phi não vêm inseridos junto a T, mas nascem em C e percolam para T. Usando Chomsky (2005) Miyagawa propõe uma tradução formal para a tipologia de Li e Thompson (1976). Segundo ele, não só traços de concordância podem percolar de $\mathrm{C}$ para $\mathrm{T}$, mas também traços discursivos de Top e Foco, havendo também línguas mistas que podem percolar tanto traços de concordância quanto traços discursivos (ex. Turco).

(11) a. $\mathrm{Cf}, \delta \rightarrow \mathrm{T} \delta \ldots$ (discourse-prominent - e.g. Japanese)

b. Cf, $\varphi \rightarrow \mathrm{T} \varphi \ldots$ (agreement-prominent - e.g. English)

Já que o PB conta tanto com o expletivo nulo (que concorda com o verbo), como com constituintes discursivos em Spec, T, poderíamos assumir que ele é uma língua mista, como o Turco. Nas construções locativas, tanto com expletivo nulo quanto com o locativo alçado, o modelo de Miyagawa funciona perfeitamente.

(12) a. Øexpl choveФ muito em São Paulo.

b. São Paulo chove $\delta$ muito.

c. Essas florestas $\Phi \boldsymbol{\delta}$ chovem muito.

A construção com expletivo nulo em (12a) teria apenas traços- $\boldsymbol{\Phi}$ i percolados para $\mathrm{T}$, requerendo a inserção de um expletivo com esses traços em seu Spec. A construção de tópico-sujeito em (12b) seria uma construção em que T teria os traços de Top para ele percolados. No exemplo (12c), porém, fica claro que o tópico pode pousar onde $\mathrm{T}$ teve Agree percolado uma vez que o tópico no plural desencadeia concordância de plural.

(13) a. Cf, $\delta, \varphi \rightarrow \mathrm{T} \delta, \varphi \ldots$ (discourse and agreement -prominent. BP)

Vejamos agora como o PE e o PB se comportam em relação a uma construção com Foco (*cf. Kato e Raposo 1996).

(14) a. Muitas mulheres amou o João.

PE

b. Muitas mulheres o João amou.

$\mathrm{PB}$

Considerando que no PE o sujeito está no interior de VP e que o Foco está em Spec, $T$, podemos dizer que o traço discursivo de Foco percola para $\mathrm{T}$, conforme previsão de Miyagawa. No PB, no entanto, o Foco (muitas mulheres) ocupa uma

${ }^{3}$ Naves, Pilati e Salles (2013) procuram aplicar a proposta de Miyagawa aos dados do PB, mas concluem que ao invés de tópicos ocuparem a posição de sujeito, o Spec,TP permanece uma posição temática, enquanto tópicos ocupam a posição periférica $\alpha \mathrm{P}$. 
posição na periferia, a que Miyagawa chama de $\alpha P$, enquanto o que percola para T são os traços de concordância $\Phi$, com o sujeito João ocupando seu Spec,T.
a. [ Muitas mulheres] amou $\delta$ João
PE
b. [Muitas mulheres] $\alpha P$ o João amou $\Phi$
PB

Comparando, portanto, o PE e o PB, verifica-se que há uma discrepância entre a percolação dos traços discursivos de tópico e de foco: O PB teria proeminência discursiva no caso do tópico, mas não quando o elemento discursivo é um Foco. $\mathrm{O} \mathrm{PE}$, por outro lado, seria uma língua de proeminência discursiva no caso do Foco, mas não no caso do Tópico.

\section{PRESSUPOSTOS TEÓRICOS}

\subsection{As questões da pesquisa}

Consideremos novamente os seguintes pares de sentença:

(16) a. Oexpl chove muito nessas florestas. PE PB

b. Estas florestas chovem muito. $\quad \mathrm{PB}$

(17) a. Øexpl furou o pneu do Hamilton. PE PB

b. O Hamilton furou o pneu. PB

As nossas questões:

a) os pares em (15) e em (16) constituem "doublets" no sentido de Kroch (1994), com as construções com expletivo nulo fadadas a desaparecer, como na hipótese de Cyrino, Duarte e Kato (2000)?

b) se as duas construções podem sobreviver por não constituírem "doublets", como derivar as construções em b.?

\subsection{Sentenças categóricas e téticas}

Para responder à pergunta sobre os dois tipos de estrutura em (16) e (17) é necessário seguir a distinção entre sentenças categóricas e téticas . Para os clássicos todas as sentenças consistem de dois atos cognitivos: um de reconhecimento de um sujeito e o outro de atribuição de um predicado ao sujeito. Para Kuroda (1972), todavia, há na verdade dois tipos de juízos: o juízo categórico e o juízo tético. O primeiro tem dois atos cognitivos, mas o segundo tem apenas um ato cognitivo, de descrever um estado ou evento, sem singularizar um sujeito ${ }^{4}$.

\footnotetext{
${ }^{4}$ No japonês uma sentença categórica tem um DP marcado - wa, enquanto a sentença tética tem um nominativo marcado com $-g a$.
} 
O que temos em (16) e (17) são dois tipos de juízos. As formas em a. são juízos téticos enquanto as formas em b. são do tipo categórico.

Para Martins (1994) as sentenças téticas no PE têm a ordem SV enquanto as categóricas têm a ordem VS. Kato (1988) mostra que com a perda da ordem VS no $\mathrm{PB}$, só com verbos inacusativos temos essa distinção. Com os demais tipos de verbo a ordem é indistintamente SV no PB

(18) Q: O que houve?

A: Chegou o avião das 10. (sentença tética) PE PB

(19) Q: E o Paulo?

A: O Paulo acabou de chegar. (sentença categórica) PE PB

(20) Q: Que foi?

A1: O Pedro viajou. $\quad$ (sentença tética) PB

(21) Q: E o Pedro?

A1. : O Pedro acaba de viajar. (sentença categórica) PE PB

Britto (2000) propõe ainda que a perda de VS no PB levou a representar sentenças categóricas como estruturas de redobro na fala, já que as téticas teriam também a ordem SV:

(22) Q: E o Pedro?

A1: O Pedro acabou de telefonar. $\quad$ (categórica: PE)

A2: O Pedro, ele acabou de telefonar. (categórica: PB)

Pode-se pressupor, então, que as formas com o expletivo nulo continuariam a permanecer como estruturas téticas no $\mathrm{PB}$ e as formas com alçamento seriam inovações no domínio das sentenças categóricas juntamente com as estruturas de redobro.

\subsection{A derivação de estruturas de redobro}

Clíticos podem aparecer em construções de deslocamento à esquerda (CLLD). Essas construções são pressupostas dentro da análise de Kayne (2001) com o clítico aparecendo no início da derivação junto com o DP associado em um "Big DP":

(23) a. Le doy un libro a Juan.

b. A Juan le doy un libro.
(Clitic doubling: CLDOUBL)

(Clitic left Dislocation: CLLD)

(24) a. Doy un libro [Juan le]

b. [Juan le] ${ }_{i}$ doy un libro $t_{i}$

c. Juan ${ }_{j}\left[t_{j} \text { le }\right]_{i}$ doy un libro $t_{i}$

d. a Juan $_{j}\left[t_{j} \text { le }\right]_{i}$ doy un libro $t_{i}$

e. $\left[\left[t_{j} \text { le }\right]_{i} \text { doy un libro } t_{i}\right]_{k}$ a Juan $t_{k}$

CLLD

CLDOUBL 


\subsection{Pronomes fracos nas estruturas de redobro}

Desde a obra seminal de Cardinalletti e Starke (1994) vimos que as línguas dispõem de pronome fortes e pronomes fracos. Modificando um pouco a proposta desses autores, incluímos nos pronomes fracos sujeito três tipos: pronomes DP como no inglês, clíticos como no Fiorentino e Afixos como no espanhol:

(25) a. Espanhol : afixo : $\left[_{\mathrm{TP}}[-\mathrm{o} \text { habla }]_{\mathrm{T} . .}\right.$

b. Fiorentino: clítico : $\left[_{\mathrm{TP}}[\text { te-parli }]_{\mathrm{T}} \ldots\right.$

c. Inglês: $\quad$ DP: $\quad\left[_{\mathrm{TP}} \mathrm{I}[\mathrm{speak}]_{\mathrm{T}} \cdots\right.$

Vamos considerar que o PE seja igual ao espanhol, enquanto o PB falado é como o Fiorentino, já que o paradigma dos pronomes sujeitos contam com pronomes fracos tipo clíticos (cf. Nunes 1990):

(26) a. Ô-vou

b. cê-vai

c. ei-vai

Abaixo teríamos o paradigma dos pronomes fracos do PE e do PB:

Tabela 2: Pronomes fracos no PE e no PB

\begin{tabular}{|c|c|c|c|c|c|c|c|c|}
\hline \multicolumn{4}{|c|}{ Pronomes fracos no PE } & & \multicolumn{4}{c|}{ Pronomes fracos no PB } \\
\hline $\begin{array}{c}\text { Nomin. } \\
\text { Sufixo }\end{array}$ & $\begin{array}{c}\text { Acus. } \\
\text { clitico }\end{array}$ & $\begin{array}{c}\text { Dat. } \\
\text { clitico }\end{array}$ & $\begin{array}{c}\text { Poss. } \\
\text { NP/DP }\end{array}$ & $\begin{array}{c}\text { Nomin. } \\
\text { clitico }\end{array}$ & $\begin{array}{c}\text { Accus. } \\
\text { clitico }\end{array}$ & $\begin{array}{c}\text { Dat. } \\
\text { Clitico }\end{array}$ & $\begin{array}{c}\text { Poss. } \\
\text { NP/ } \\
\text { DP }\end{array}$ \\
\hline- o & me & me & meu & & o- & me & me & meu \\
\hline$-\mathrm{s}$ & te & te & teu & & cê- & te & te & teu/seu \\
\hline$-\varnothing$ & o/lo & lhe & seu & & ei- & $\varnothing$ & $\varnothing$ & $\varnothing$ \\
\hline
\end{tabular}

Na escrita do PB pronomes fortes e fracos aparecem grafados como fortes. ${ }^{5}$ Mas na fala a distribuição fica clara nas estruturas de redobro:

(27) a. Cê tá louco!

b. Você, cê tá louco!

c. *Cê, cê tá louco.

d. *Cê, você tá louco.
PB

$\mathrm{PB}$

$\mathrm{PB}$

PB

${ }^{5}$ Foi esse o motivo que levou Kato (1999) a achar que os pronomes fracos sujeitos do PB eram do tipo do inglês e do alemão: livres. As estruturas de redobro nos mostram, contudo, que nossos pronomes fracos sujeito são clíticos. 
Nem todas as línguas têm o pronome forte na forma nominativa:

(28) a. Me, I love the Beatles.

b. Moi, j'áime les Beatles.

\subsection{A análise das estruturas de redobro no PB}

Adotamos aqui a proposta de redobro ( Clitic Doubling) de Kayne, partindo da derivação de um "Big DP" e usando a teoria de movimento por cópia (Nunes 2004).

(29) O Pedro, [ei] acaba de ligar.

(30) a. $\left[\left[_{\mathrm{VP}}[\text { ei- o Pedro }]_{\mathrm{DP}}\right.\right.$ acaba de ligar $]$

-subida do verbo para $\mathrm{T}$ e alçamento do clítico ei para adjunção a $\mathrm{T}$, onde tem caso nominativo e concordância checados

b. $\left[_{\mathrm{TP}}\left[\mathrm{ei}_{\mathrm{i}} \text {-acaba }\right]_{\mathrm{T}}\left[_{\mathrm{VP}}\left[\right.\right.\right.$ ei $\mathrm{i}_{\mathrm{i}}$ - o Pedro] acaba $_{\mathrm{V}}$ de ligar $]$

- alçamento de o Pedro para Spec,T, para checar o traço D de T

c. $\left[{ }_{\mathrm{TP}}\right.$ o Pedro ${ }_{\mathrm{j}}\left[\mathrm{ei}_{\mathrm{i}}-\mathrm{acaba}\right]_{\mathrm{T}}\left[_{\mathrm{VP}} \mathrm{ei}_{\mathrm{i}}-\mathrm{o}\right.$ Pedro $\left._{\mathrm{j}}\right]$ acaba $_{\mathrm{V}}$ de ligar $]$

FF: d. $\left[_{T P}\right.$ o Pedro $\left[e_{i}-\text { acaba }\right]_{T}\left[{ }_{V P} e_{i}-0\right.$ Pedro $\left._{j}\right]$ aeaba $_{\forall}$ de ligar $]$

\section{ANÁLISE DAS CONSTRUÇÕES DE TÓPICO-SUJEITO}

As construções de tópico-sujeito, assim como as de redobro do sujeito, são sentenças categóricas e não constituem "doublets" das sentenças com expletivo nulo, que são téticas.

As construções de tópico-sujeito são evoluções a partir de Deslocamento Clítico à Esquerda (CLLD), ou de DE (Deslocamento à Esquerda), que também são sentenças categóricas.

A mudança de CLLD para construções de tópico-sujeito tiveram o seu "trigger" na mudança morfológica no paradigma dos pronomes fracos. O clítico locativo $h i$ do Português Antigo e Medieval foi o primeiro a zerar, até mesmo no $\mathrm{PE}^{6}$ :

$\begin{array}{lcllllll}(31) & \text { PE } & & & & \text { PB } & & \\ \text { Acus. } & \text { Dat. } & \text { Poss. } & \text { Locat. } & \text { Acus } & \text { Dat } & \text { Poss } & \text { Locat } \\ \text { me } & \text { me } & \text { meu } & & \text { me } & \text { me } & \text { meu } & \\ \text { te } & \text { te } & \text { teu } & & \text { te } & \text { te } & \text { teu } & \\ \text { o/lo } & \text { lhe } & \text { seu/ } \varnothing & \varnothing & \varnothing & \varnothing & \text { seu/ } \varnothing & \varnothing\end{array}$

Vejamos primeiramente a derivação de construções de CLLD comum até o século XIX e existentes ainda no PE.

${ }^{6} \mathrm{O}$ possessivo [-animado] é nulo. 
- CLLD a partir de dativo

(32) Ao meu time faltou-lhe sorte. PE

A derivação de um CLLD se inicia com o constituinte deslocado e o clítico formando um "big DP" :

(33) a. [o meu time lhe $]_{\mathrm{DP}}$ compondo o VP

b. $\left[{ }_{\mathrm{VP}}\right.$ faltou sorte $\left.[\mathrm{o} \text { meu time lhe }]_{\mathrm{DP}}\right]$ subida do verbo para $T$

c. faltou $\left[_{\mathrm{VP}}\right.$ faltou sorte $\left.[\mathrm{o} \text { meu time } \mathrm{lhe}]_{\mathrm{DP}}\right] \quad$ clitic climbing

d. faltou-lhe $\left.\left[_{\mathrm{VP}} \text { faltou sorte [o meu time lhe }\right]_{\mathrm{DP}}\right] E P P\left(\right.$ inserção do $\left.\varnothing_{\text {expl }}\right)$

e. $\left[\varnothing_{\text {expl }} \text { faltou-lhe }\left[_{\mathrm{VP}} \text { faltou sorte [o meu time lhe }\right]_{\mathrm{DP}}\right]_{\mathrm{TP}}$ topicalização

f. $\left[_{\mathrm{TopP}} \mathrm{O}\right.$ meu time $\left[\varnothing_{\text {expl }}\right.$ faltou-lhe $\left.\left[_{\mathrm{VP}} \text { faltou sorte [ao meu time lhe }\right]_{\mathrm{DP}}\right]$ inserção de preposição

g. $\left[_{\mathrm{TopP}}\right.$ ao meu time $\left[\varnothing_{\text {expl }}\right.$ faltou-lhe $\left[_{\mathrm{VP}}\right.$ faltou sorte $\left.[\mathrm{o} \text { meu time lhe }]_{\mathrm{DP}}\right]$

FF: $\quad$ g. $\left[_{\text {TopP }}\right.$ ao meu time $\left.\left[\varnothing_{\text {expl }} \text { faltou-lhe [ faltou_sorte [o meu time the }\right]_{\mathrm{DP}}\right]$

Vejamos agora a derivação de uma estrutura de tópic-sujeito.

(34) O meu time faltou sorte. PB

A derivação de uma construção de tópico-sujeito como (33) começa com o sujeito/tópico nascendo também no interior de um "Big DP", porém associado a um clítico nulo, sem caso (v. Tabela 2);

$\mathrm{O}$ associado do clítico nulo se move para Spec, $\mathrm{T}$, onde ele checa nominativo e concordância (os traços-phi e o traço D).

(35) a. [o meu time Ø]

b. $\left[{ }_{\mathrm{VP}}\right.$ faltou sorte $\left.[\mathrm{o} \text { meu time } \varnothing]_{\mathrm{DP}}\right]$

c. faltou $\left.\left[_{\mathrm{VP}} \text { faltou sorte [o meu time } \varnothing\right]_{\mathrm{DP}}\right]$

d. Ø-faltou $\left.\left[_{\mathrm{VP}} \text { faltou sorte [o meu time } \varnothing\right]_{\mathrm{DP}}\right]$

e. $\left.\left[{ }_{\mathrm{TP}} \text { o meu time } \varnothing \text {-faltou [ }{ }_{\mathrm{VP}} \text { faltou sorte [o meu time } \varnothing\right]_{\mathrm{DP}}\right]$ derivação do VP

subida do verbo para $T$

clitic-climbing

$E P P$

- A derivação de um tópico-sujeito a partir de um possessivo:

(36) O Hamilton furou um pneu.

(37) a. [o Hamilton Ø]

b. furou um pneu [o Hamilton $\varnothing]$

c. furou um Ø-pneu [ o Hamilton Ø]

subida do possessivo

d. $\left[_{T P} \mathrm{O}\right.$ Hamilton furou um $\varnothing$-pneu [ o Hamilton $\left.\left.\varnothing\right]\right]$

Para derivar o CLLD a partir do genitivo seu, em lugar de EPP, o Hamilton sobe para a posição deslocada e pode opcionalmente receber a preposição de. 
(38) (D)o Hamilton, furou um pneu seu.

- Deslocamento ou tópico/sujeito a partir do locativo nulo (hi): (PE e PB)

No PA quando um PP aparecia como locativo, ele era frequentemente redobrado pelo clítico locativo hi ( Castilho 2007)

(39) a. e que estava [hy na corte] hũũ filho dessa dona Timbor.

b. [enna corte] [ hi estava Dom Galaaz.

Vamos assumir que no PE e PB modernos temos ainda um clítico nulo locativo que pode aparecer também em estruturas de redobro.

(40) a. Chove [ (hi) em São Paulo]

b. ((Em) São Paulo, (hi) chove.

A sentença seguinte, possível em PE e PB é ambígua entre as estruturas de Deslocamento, sem preposição, e a de tópico/sujeito.

(41) São Paulo chove.

Minha proposta é que, enquanto no PE temos São Paulo deslocado, no PB temos um tópico sujeito em Spec,T. A confirmação dessa conjectura está na sentença (42), possível em $\mathrm{PB}$, mas não em PE.

$\begin{array}{ll}\text { (42) a. Essas florestas chovem muito. } & \text { PB *PE } \\ \text { b. (N)essas florestas chove muito. } & \text { PE PB }\end{array}$

\section{CONCLUSÕES}

- As construções de tópico-sujeito no PB, inexistentes no PE, evoluíram das sentenças categóricas de CLLD do português do século XIX, tendo tido como gatilho a mudança morfológica no sistema de clíticos e pronomes possessivos, confirmando a conjectura na linha de Borer$\mathrm{Chomsky}^{7}$, de atribuir às distinções morfológicas as mudanças de parâmetro.

- Embora o PB venha perdendo o sujeito nulo, não há previsão, na minha análise, de sentenças com expletivo nulo desaparecerem, já que elas não competem como "doublets" das sentenças com tópico-sujeito.

- Assumindo que os clíticos nulos não têm caso, seus associados podem se mover para posições onde recebem/ checam nominativo, a saber,

\footnotetext{
${ }^{7}$ É a linha que atribui a variação paramétrica a itens lexicais, ou à morfologia, proposta primeiro em Borer (1984) e depois assumida em Chomsky (1995).
} 
Spec,T ou na periferia à esquerda, onde podem receber caso de uma preposição ou receber o caso nominativo 'default'.

- O clítico locativo, embora desaparecido desde o português clássico, ainda tem presença nas derivações de sentenças com tópico-sujeito e nas de deslocamento clítico.

\section{REFERÊNCIAS BIBLIOGRÁFICAS}

ANDRADE BERLINCK, R. (1989). La Position du Sujet en Portugais: étude classes. In: van Riemsdijk (org.). Clitics in the Languages of Europe. Walter de Gruyter.

BORER, H. (1984). Parametric Syntax: Case studies in Semitic and Romance languages. Dordrecht: Foris.

BRITTO, H. (2000). Syntactic codification of categorical and thetic judgments in Brazilian Portuguese. In: M. A. Kato \& E. V. Negrão (orgs.). 195-223.

CASTILHO, C. M. M. (2007). A saga do locativo hi redobrado no português medieval. In: A.T. de Castilho, M. A.Torres-Moraes, R. E. V. Lopes \& S. M. L. Cyrino (orgs.). Descrição, História e Aquisição do Português Brasileiro. Campinas: Ed. Pontes/ FAPESP.

CHOMSKY, N. (1995). The Minimalist Program. Cambridge, Mass.: MIT Press.

CHOMSKY, N. (2005). Three factors in language design. Linguistic Inquiry. 36, 1-23.

CYRINO, S. M. L. (1993). Observaçoes sobre a mudança diacônica no portugês do Brasil: objeto nulo e clíticos. In: I. Roberts e M. A. Kato (orgs.). 163-84.

CYRINO, S. M. L., DUARTE, M. E. L. e KATO, M. A. (2000). Visible subjects and invisible clitics in Brazilian Portuguese. In: M. A. Kato \& E. V. Negrão (orgs.). 55-104.

DUARTE, M. E. L.(1993). Do pronome nulo ao pronome pleno: a trajetória do sujeito no português do Brasil. In: I. Roberts e M. A. Kato (orgs.). 107-128.

DUARTE, M. E. L. (1995). A perda do princípio "evite pronome" no português brasileiro. Campinas, 1995. Tese (doutorado em Linguística), Unicamp.

KATO, M. A. (1989). Tópico e sujeito: duas categorias na sintaxe?“. Cadernos de Estudos Linguísticos (17), 109-131.

KATO, M. A. (1999). Strong and Weak Pronominals and the Null Subject Parameter. Probus, 11, 1, 1999, 1-31.

KATO, M. A. (2012). O Português são dois...ou três?. In T. Lobo, Z. Carneiro, S. Ribeiro, J. Soledade, A. Alemida (orgs). ROSAE-Linguística histórica, história das línguas e outras histórias. 114131. Salvador: EDUFBA. 2012.

KATO, M. A. e DUARTE, M. E. L. (2005). (Micro)parametric variation between European (EP) and Brazilian Portuguese (BP): similarities and differences with ongoing changes in Latin American Spanish. Comunicação apresentada no XIV Congresso Internacional da Alfal (Workshop: I România Nova). Monterrey. 
KATO, M. A. e DUARTE, M. E. L. (2008). Mudança paramétrica e orientação para o discurso. Trabalho apresentado no XXIV Encontro Nacional da Associação Portuguesa de Linguística, Braga.

KATO, M. A. e DUARTE, M. E. L. (2014). A variação entre construções finitas pessoais e impessoais no Português Brasileiro. Sociodialeto, vol 4, Edição 12, 153-177.

KATO M. A., DUARTE, M. E. L., CYRINO, SONIA e BERLINCK, ROSANE de A. (2006). Portugês brasileiro no fim do século XIX e na virada do milênio. In: S. A. M. Cardoso, J. A. Mota, R. V. Mattos e Silva (orgs.). Quinhentos Anos de História Lingüistica do Brasil. Salvador, Fundo de Cultura da Bahia. 413-438.

KATO, M. A. e NEGRÃO, E. V. (2000). Brazilian Portuguese and the Null Subject Parameter. Frankfurt: Vervuert/Iberoamericana.

KATO, M. A. e RAPOSO. E. (1996). European and Brazilian word order: questions, focus and topic constructions. In C. Parodi, A. C. Quicoli, M. Saltarelli \& M. L. Zubizarreta (orgs.). Aspects of Romance Linguistics. Washington: Georgetown U. Press, 267-277.

KATO, M. A. e RAPOSO. E. (2007). Topicalization in European and Brazilian Portuguese. In: Camacho, J. et al. (orgs.). Romance Linguistics: Selected Papers from the $36^{\text {th }}$ Linguistic Symposium on Romance Languages (LSRL). Amsterdã: John Benjamins Publishing Company, 213-26.

KATO, M. A. e TARALLO, F. (2003). The Loss of vs syntax in Brazilian Portuguese. In: B. SCHLIEBE-LANGE, KOCH, I., JUNGBLUTH, K. (orgs.). Dialogue between Schools: Sociolinguistics, Conversational Analysis and GenerativeTheory in Brazil. Munique: Nodus Publicationen, Klaus D. Ditz, 101-29.

KAYNE, R. (1989). Null subjects and clitic climbing. In: O. Jaeggli e K Safir (orgs.). The Null Subject Parmeter. Dordrecht: Kluwer. 239-61.

KAYNE, R. (2001). Pronouns and their antecedents. In S. Epstein and D. Seely (orgs.). Derivation and Explanation in the Minimalist Program, Malden, Mass:Blackwell. 133-66.

KISS, K. (1995). Discourse Configurational Languages. New York/Oxford: Oxford University Press.

KROCH, A. (1994). Morphosyntactic variation. In: Beals et al (orgs.). Proceedings of the Thirtieth Annual Meeting of the Chicago Linguistic Society. Vol 2 . 180-201.

KURODA, Y. (1972). The concept of Subject in Grammar. In: SHBATANI, M. (ed.). Sintax and Semantics: Japanese Generative Grammar. Nova York: Academic Press, 1-16.

LI, C. N. e THOMPSON, S. (1976). Subject and Topic: A New Typology of Language. In: LI, C. N. (org.). Subject and Topic. Nova York: Academic Press Inc. 457-490.

MARTINS, A. M. (1994). Clíticos na História do Português.Universidade de Lisboa: Tese de Doutorado.

MIYAGAWA, S. (2007).Unifying agreement and agreement-less languages. Proceedings of WAFL2, pp. 47-66. MIT Working Papers in Linguistics, 54.

MIYAGAWA, S. (2010). Why agree? Why move? Unifying agreement-based and discourse configurational languages. Linguistic Inquiry Monograph 54, MIT Press. 
NAVES, R. R., PILATI, E. S. e SALLES. H. (2013). The properties of INFL in the context of parametric variation between PB and PE: and analysis in terms of feature inheritance from C to T. Trabalho apresentado no IV Workshop Romania Nova (VIII Congresso Internacional da ABRALIN), Natal.

NEGRÃO, E. V. (1999). O Português brasileiro: Uma língua voltada para o discurso. USP: Tese de Livre-docência.

NUNES, J. (1990). Nominative pronouns reduction in Brazilian Portuguese. University of Maryland. Ms.

NUNES, J. (2004). Linearization of chains and Sideward Movement. Cambridge, Mass: The MIT Press.

PAGOTTO, E. (1993). Clíticos, mudança e seleçãoo natural. In: I. Roberts e M. A. Kato (orgs.). 185206.

PONTES, E. (1987). O Tópico no Português do Brasil. Campinas: Pontes.

ROBERTS, I. e KATO, M. A. (1993). Português Brasileiro: uma viagem diacrônica. Campinas: Editora de UNICAMP.

TORIBIO, J. (1996). Dialectal variation in the licensing of null referential and expletive subjects. In: C. Parodi, C. Quicoli, M. Saltarelli \& M. L. Zubizarreta (orgs.). Aspects of Romance Linguistics. Washington, DC: Georgetown University Press. 409-32. 